\title{
Strong evidence for LncRNA ZNRD1-AS1, and its functional Cis-eQTL locus contributing more to the susceptibility of lung cancer
}

\author{
Dan Li ${ }^{1, *}$, Lei Song ${ }^{1, *}$, Zhongmei Wen ${ }^{1}$, Xiaoping Li $^{2}$, Jing Jie ${ }^{1}$, Yan Wang ${ }^{1}$, Liping Peng ${ }^{1}$ \\ ${ }^{1}$ Department of Respiratory Medicine, The First Hospital of Jilin University, Changchun, China \\ ${ }^{2}$ Department of Pediatrics, The First Hospital of Jilin University, Changchun, China \\ "These authors contributed equally to this work
}

Correspondence to: Liping Peng, email: plp640317@163.com

Keywords: lung cancer, LnCRNA, ZNRD1-AS1, SNP, susceptibility

Received: October 26, $2015 \quad$ Accepted: April 19, $2016 \quad$ Published: April 29, 2016

\section{ABSTRACT}

Long noncoding RNAs (IncRNAs), involved in cancer biology, contributing to essential cancer cell functions such as proliferation, invasion, and metastasis, have received increasing attention recently. Human Zinc ribbon domain containing 1 (ZNRD1) has been confirmed to be involved in carcinogenesis and development of multiple cancers. ZNRD1-AS1, a IncRNA in the upstream region of ZNRD1 which could down-regulate the expression of ZNRD1, has been identified as a possible component in carcinogenesis. The underlying relations of ZNRD1-AS1 with lung cancer development and metastasis remain obscure. In current study, we first evaluated the expression ZNRD1-AS1 and ZNRD1 among lung cancer tissues and corresponding normal tissues, which showed higher expression of ZNRD1-AS1 and lower expression of ZNRD1. To reveal the underlying mechanisms, we then investigated the associations between ZNRD1 eQTLs SNPs in ZNRD1-AS1 and risk of lung cancer in Han Chinese populations. G allele of SNP rs9261204 was significantly associated with an increased risk of lung cancer when compared with $A$ allele (OR: 1.45; 95\% CI: 1.19-1.75; $P=1.06 \times 10^{-4}$ ). A weaker, but similar effect was also observed in bladder cancer. SNP rs3757328 was also associated with increased risk of lung cancer (OR: 1.34; 95\% CI: 1.07-1.67; $P=0.011$ ). Our findings first confirmed the contribution of LnCRNA ZNRD1-AS1 to the development of lung cancer in Asian population.

\section{INTRODUCTION}

Zinc ribbon domain containing 1 (ZNRD1) has been confirmed to be involved in carcinogenesis and development of multiple cancers, including gastric cancer, leukemia, esophageal squamous cell carcinoma, renal cell carcinoma, cervical cancer and hepatocellular carcinoma [1-8]. Long non-coding RNAs (LncRNAs) has been confirmed to be associated with susceptibility, invasion, metastasis, and overall and disease-free survival of many cancers $[9,10]$. Recently, two studies also evaluated the association of expression quantitative trait loci (eQTL) in LncRNA ZNRD1-AS1, a LncRNA in the upper region of ZNRD1 gene which could downregulate the gene expression of ZNRD1, with the susceptibility and development of cervical cancer and hepatocellular carcinoma $[6,7]$. However, no association study has conducted to evaluate the relationship between gene expression of ZNRD1 and lung cancer up to present, either in vivo or in vitro studies. Basing on the clues above, we hypothesized that: (1) expression of ZNRD1AS1 was up-regulated and expression of ZNRD1 was down-regulated in lung cancer tissues; (2) Cis-eQTL loci in LncRNA ZNRD1-AS1 (rs3757328, rs6940552, and rs9261204) contribute to the development of lung cancer. Thus, we conducted this study to test the hypotheses. 


\section{RESULTS}

In current study, we first evaluated the expression levels of ZNRD1-AS1 and ZNRD1 among 20 lung cancer tissues and corresponding normal tissues using Quantitative (real-time) PCR. As shown in Figure 1, the results showed that expression of ZNRD1-AS1 was up-regulated and expression of ZNRD1 was down-regulated in lung cancer tissues (both $P$ value $<0.0001$ ). Taken the results above together, these findings suggest a tumor suppressor function for ZNRD1 gene and a tumor contributor function for
LncRNA ZNRD1-AS1in the process of carcinogenesis of lung cancer. A weaker, but similar effect was also observed in bladder cancer (Figure 1).

We evaluated the associations of three tagSNPs of the Cis- eQTL for ZNRD1 in ZNRD1-AS1 gene region (rs3757328, rs6940552, and rs9261204) with risk of lung cancer and bladder cancer. As shown in Table 1, the distributions of SNP rs3757328, SNP rs9261204 and SNP rs6940552 in lung cancer and healthy control subjects were displayed. The results showed that compared with A allele, $\mathrm{G}$ allele of rs9261204 was significantly associated

\section{ZNRD1}

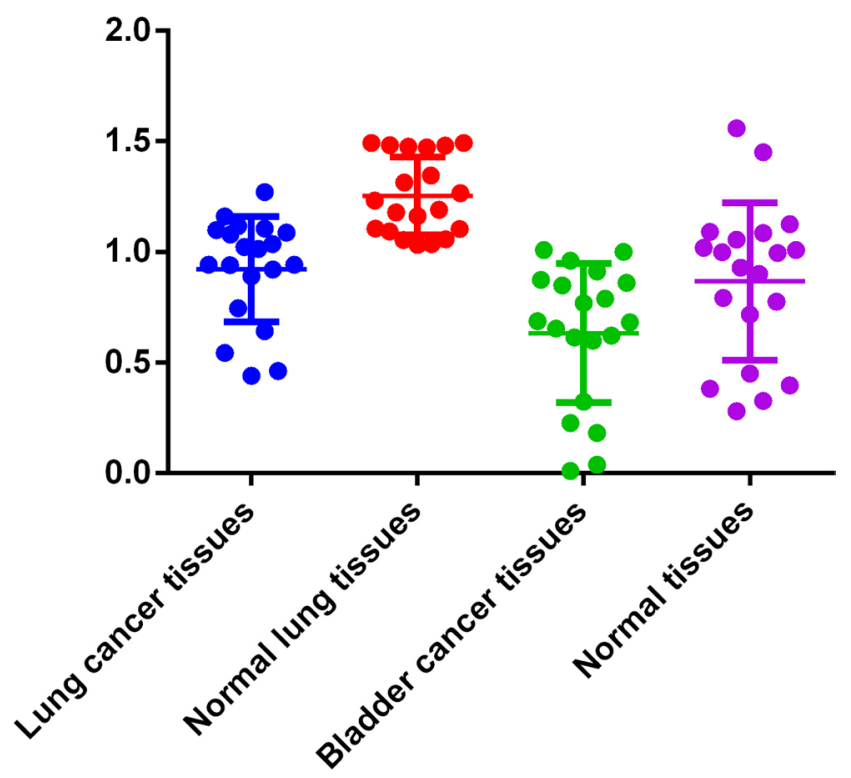

ZNRD1-AS1

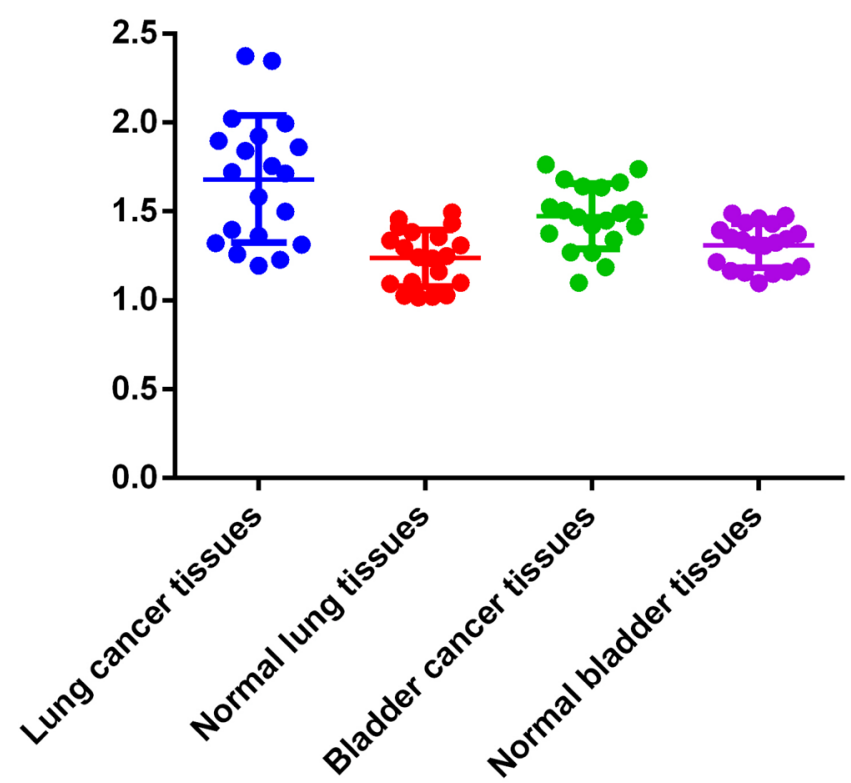

Figure 1: Quantification and statistical analysis of ZNRD1-AS1 and ZNRD1 expression in bladder cancer and matched normal tissues 
Table 1: Association between 3 eQTLs SNPs in ZNRD1-AS1 and bladder cancer susceptibility

\begin{tabular}{|c|c|c|c|c|c|}
\hline \multirow[b]{2}{*}{ Genotype } & \multirow[b]{2}{*}{ Controls } & \multicolumn{2}{|c|}{ Lung cancer } & \multicolumn{2}{|c|}{ Bladder cancer } \\
\hline & & Cases & $\begin{array}{l}\text { Adjusted OR } \\
(95 \% \mathrm{CI})^{*}\end{array}$ & Cases & $\begin{array}{l}\text { Adjusted OR } \\
(95 \% \mathrm{CI})^{*}\end{array}$ \\
\hline \multicolumn{6}{|l|}{ rs 3757328} \\
\hline GG & 340 & 305 & 1.00 (reference) & 337 & 1.00 (reference) \\
\hline $\mathrm{AG}$ & 150 & 175 & $1.30(0.99-1.70)$ & 146 & $0.98(0.74-1.29)$ \\
\hline $\mathrm{AA}$ & 10 & 20 & $2.22(1.05--4.75)$ & 17 & $1.71(0.78-3.77)$ \\
\hline Additive & & & $1.34(1.07-1.67)$ & & $1.07(0.85-1.35)$ \\
\hline $\mathrm{P}$ trend & & & 0.011 & & 0.556 \\
\hline \multicolumn{6}{|l|}{ rs6940552 } \\
\hline$\overline{\mathrm{GG}}$ & 289 & 265 & 1.00 (reference) & 274 & 1.00 (reference) \\
\hline $\mathrm{AG}$ & 169 & 179 & $1.15(0.88-1.51)$ & 175 & $1.09(0.84-1.43)$ \\
\hline $\mathrm{AA}$ & 42 & 56 & $1.45(0.94-2.23)$ & 51 & $1.28(0.82-1.98)$ \\
\hline Additive & & & $1.21(0.99-1.47)$ & & $1.13(0.93-1.38)$ \\
\hline $\mathrm{P}$ trend & & & 0.056 & & 0.224 \\
\hline \multicolumn{6}{|l|}{ rs9261204 } \\
\hline $\mathrm{AA}$ & 280 & 228 & 1.00 (reference) & 241 & 1.00 (reference) \\
\hline $\mathrm{AG}$ & 180 & 207 & $1.41(1.08-1.84)$ & 200 & $1.29(0.99-1.68)$ \\
\hline GG & 40 & 65 & $1.99(1.30-3.05)$ & 59 & $1.71(1.11-2.64)$ \\
\hline Additive & & & $1.45(1.19-1.75)$ & & $1.33(1.09-1.61)$ \\
\hline $\mathrm{P}$ trend & & & $1.06 \times 10^{-4}$ & & 0.004 \\
\hline
\end{tabular}

*Asjusting for age, gender, smoking status, and drinking status.

with 1.45-fold risk of lung cancer (OR: 1.45; 95\% CI: $\left.1.19-1.75 ; P=1.06 \times 10^{-4}\right)$, as well as bladder cancer (OR: 1.33 ; 95\% CI: $1.09-1.61 ; P=0.004)$. Per the UCSC Genome Browser, SNP rs9261204 falls within several transcription factor ChIP regions including CTCF, SMC3, RAD21, ZNF143, TEAD4, PBX3, AND FOXA1. Besides, rs3757328 was also associated with 1.34 -fold risk of lung cancer (OR: 1.34; 95\% CI: $1.07-1.67 ; P=0.011$ ). Further, rs6940552 showed an marginal association with lung cancer risk (OR: 1.21; 95\% CI: 0.99-1.47; $P=0.056)$. Overall, our results provide strong evidence that LncRNA ZNRD1-AS1, and its functional Cis-eQTL locus contribute more to the susceptibility of lung cancer.

\section{DISCUSSION}

In current study, we first examined the gene expression of LncRNA ZNRD1-AS1 and ZNRD1 in lung cancer tissues and corresponding normal tissues; then we investigated whether eQTLs SNPs located in ZNRD1-AS1 and lung cancer risk in Han Chinese populations. Results showed expression of ZNRD1-AS1 was up-regulated and expression of ZNRD1 was down-regulated in lung cancer tissues, and G allele of SNP rs9261204 was significantly associated with lung cancer risk when compared with A allele. Furthermore, a weaker, but similar effect was also observed in bladder cancer. This should be the initial study which aims to explore the relationship between LncRNA ZNRD1-AS1, its variants, and ZNRD1 and development of lung cancer and bladder cancer.

ZNRD1 was downegulated in gastric cancer tissues, compared with adjacent normal tissues [11]. Through transfecting siRNA vectors of ZNRD1 and ZNRD1 cDNA to normal gastric cells or gastric cancer cells, significantly quicker proliferation, and decreased growth rate were detected [11]. In current study, we also found the decreased expression levels of ZNRD1 in the lung cancer tissues, compared with corresponding normal tissues. ZNRD1 spans the HLA region spanning and many studies have suggested that genetic variants of the genes in HLA region contributed to the risk of bladder cancer [12-15]. These evidence above suggested the potential suppressor role of ZNRD1 in carcinogenesis.

IncRNAs, contributing to cancer cell functions including proliferation, apoptosis, invasion, and metastasis, have received increasing attention recently [16-18]. LncRNA ZNRD1-AS1 was located in the upper region of ZNRD1. Wen et al. [7] first reported that rs9261204 
and rs6940552 in lncRNA ZNRD1-AS1 may influence not only chronic HBV infection, but also hepatocellular carcinoma (HCC) development. The results of genetic associated study showed that rs3757328 were statistically associated with the lower gene expression of lncRNA ZNRD1 and high gene expression of ZNRD1-AS1 [7]. In current study, SNP rs9261204 was significantly associated with lung cancer and bladder cancer risk. Conclusively, this study showed that LncRNA ZNRD1-AS1, its variants, and ZNRD1 contribute more to the development of lung cancer from different approaches, including in vivo, and epidemiological investigations.

\section{MATERIALS AND METHODS}

\section{Study group and personal interview}

Included in this study were 500 newly diagnosed with histologically confirmed lung cancer cases, and 500 controls which were identified among residents of the cases' neighborhoods of residence and matched to cases by gender, race/ethnicity and age ( \pm 5 years). The exclusion factors for cases included previous cancer, metastasized cancer from other or unknown origin, and previous radiotherapy or chemotherapy. We also replicated the associations among 500 bladder cancer cases. In-person questionnaires administered to all study participants were used to collect demographic variables. A $4 \mathrm{~mL}$ peripheral venous blood specimens were collected at the time of interview. Informed consent was obtained from each participant and all procedures and study guidelines were approved by the ethics Committee.

\section{RNA isolation and qRT-PCR}

Total RNA was extracted from cancer tissue samples using TRIzol (Invitrogen, Carlsbad, CA) according to the manufacturer's instructions. The expression levels of ZNRD1AS1 and ZNRD1 among cancer tissues and corresponding normal tissues were detected using Quantitative (realtime) PCR performed with SYBR Green 2x Master Mix (Life Technologies Carlsbad, CA, USA). Delta CTs were normalized to GAPDH reference gene, and $\triangle \triangle C T$ analysis was performed to calculate relative expression of RNA.

\section{Determination of eQTL and genotyping}

Using database RegulomeDB [19], which guides interpretation of regulatory variants in the human genome, we selected rs3757328, rs6940552, and rs9261204 as the tagSNPs of the Cis- eQTL for ZNRD1 in ZNRD1AS1 gene region as previously [6, 7]. The genotyping were determined by using a polymerase chain reactionrestriction fragment length polymorphism method (PCRRFLP). To confirm the genotyping results, PCR-amplified DNA samples were examined by DNA sequencing, and the results were $100 \%$ concordant.

\section{Statistical analyses}

First, the expression levels of ZNRD1-AS1 and ZNRD1 between matched pairs of samples were analyzed using Wilcoxon matched-pairs signed rank test. Then, SNP-level association tests for the identified tagSNPs were conducted using unconditional logistic regression treating alternate alleles as an ordinal variable (a logadditive model). Haploview was employed to analyze linkage disequilibrium (LD) parameters ( $D^{\prime}$ and r2). Statistical analyses were carried out using SAS (Version 9.3; SAS Inc.). $P<0.05$ in a two-sided test was considered statistically significant.

\section{ACKNOWLEDGMENTS AND FUNDING}

We thank all participants who take part in this study.

\section{CONFLICTS OF INTEREST}

The authors declare that they have no conflicts of interest.

\section{REFERENCES}

1. Zhao Y, Hong L, Wang R, Fan D. Expression and prognostic value of ZNRD1 in esophageal squamous cell carcinoma. Dig Dis Sci. 2009; 54:586-592.

2. Guo W, Zhao YP, Jiang YG, Wang RW, Hong L, Fan DM. ZNRD1 might mediate UV irradiation related DNA damage and repair in human esophageal cancer cells by regulation of ERCC1. Dis Esophagus. 2008; 21:730-736.

3. Hong L, Piao Y, Han Y, Wang J, Zhang X, Du Y, Cao S, Qiao T, Chen Z, Fan D. Zinc ribbon domain-containing 1 (ZNRD1) mediates multidrug resistance of leukemia cells through regulation of P-glycoprotein and Bcl-2. Mol Cancer Ther. 2005; 4:1936-1942.

4. Zhang YM, Zhao YQ, Pan YL, Shi YQ, Jin XH, Yi H, Fan DM. Effect of ZNRD1 gene antisense RNA on drug resistant gastric cancer cells. World J Gastroenterol. 2003; 9:894-898.

5. Ding F, Xin DQ, Pan BN. [Expression of ZNRD1 protein in human renal cell carcinoma]. Zhonghua Zhong Liu Za Zhi. 2008; 30:821-824.

6. Guo L, Wen J, Han J, Jiang J, Xie S, Feng X, Wei B, Dai J, Zhang K, Qi J, Ma H, Shi J, Ren J, et al. Expression quantitative trait loci in long non-coding RNA ZNRD1-AS1 influence cervical cancer development. Am J Cancer Res. 2015; 5:2301-2307.

7. Wen J, Liu Y, Liu J, Liu L, Song C, Han J, Zhu L, Wang C, Chen J, Zhai X, Shen H, Hu Z. Expression quantitative trait loci in long non-coding RNA ZNRD1-AS1 influence both HBV infection and hepatocellular carcinoma development. Mol Carcinog. 2015; 54:1275-1282. 
8. Shang Y, Zhang Z, Liu Z, Feng B, Ren G, Li K, Zhou L, Sun Y, Li M, Zhou J, An Y, Wu K, Nie Y, Fan D. miR-508-5p regulates multidrug resistance of gastric cancer by targeting ABCB1 and ZNRD1. Oncogene. 2014; 33:3267-3276.

9. Li J, Han L, Roebuck P, Diao L, Liu L, Yuan Y, Weinstein JN, Liang H. TANRIC: An Interactive Open Platform to Explore the Function of IncRNAs in Cancer. Cancer Res. 2015; 75:3728-3737.

10. Zhao W, An Y, Liang Y, Xie XW. Role of HOTAIR long noncoding RNA in metastatic progression of lung cancer. Eur Rev Med Pharmaco. 2014; 18:1930-1936.

11. Hong L, Zhang Y, Liu N, Liu C, Zhi M, Pan Y, Lan M, Sun L, Fan D. Suppression of the cell proliferation in stomach cancer cells by the ZNRD1 gene. Biochem Biophys Res Commun. 2004; 321:611-616.

12. Middleton D, Vilchez JR, Cabrera T, Meenagh A, Williams F, Halfpenny I, Maleno I, Ruiz-Cabello F, LopezNevot MA, Garrido F. Analysis of KIR gene frequencies in HLA class I characterised bladder, colorectal and laryngeal tumours. Tissue Antigens. 2007; 69:220-226.

13. El-Chennawi FA, Auf FA, El-Diasty AM, El-Daim MA, El-Sherbiny SM, Ali A, El-Baz M, El-Hameed MA, Paul P, Ibrahim EC, Carosella E, Ghoneim MA. Expression of HLA-G in cancer bladder. Egyp J Imm. 2005; 12:57-64.

14. Maleno I, Romero JM, Cabrera T, Paco L, Aptsiauri N, Cozar JM, Tallada M, Lopez-Nevot MA, Garrido F. LOH at 6 p21.3 region and HLA class I altered phenotypes in bladder carcinomas. Immunogenetics. 2006; 58:503-510.

15. Braf ZF, Gazit E, Many M. HLA-A and HLA-B antigens in transitional cell carcinoma of the bladder. J Urol. 1979; 122:465-466.

16. Zhao F, Lin T, He W, Han J, Zhu D, Hu K, Li W, Zheng Z, Huang J, Xie W. Knockdown of a novel lincRNA AATBC suppresses proliferation and induces apoptosis in bladder cancer. Oncotarget. 2015; 6:1064-1078. doi: 10.18632/ oncotarget.2833.

17. Tsai MC, Manor O, Wan Y, Mosammaparast N, Wang JK, Lan F, Shi Y, Segal E, Chang HY. Long noncoding RNA as modular scaffold of histone modification complexes. Science. 2010; 329:689-693.

18. Gupta RA, Shah N, Wang KC, Kim J, Horlings HM, Wong DJ, Tsai MC, Hung T, Argani P, Rinn JL, Wang Y, Brzoska P, Kong B, et al. Long non-coding RNA HOTAIR reprograms chromatin state to promote cancer metastasis. Nature. 2010; 464:1071-1076.

19. Boyle AP, Hong EL, Hariharan M, Cheng Y, Schaub MA, Kasowski M, Karczewski KJ, Park J, Hitz BC, Weng S, Cherry JM, Snyder M. Annotation of functional variation in personal genomes using RegulomeDB. Genome Res. 2012; 22:1790-1797. 